\title{
Prevention of chemotherapy- and radiotherapy-induced nausea and vomiting: Guideline update and results of the Perugia consensus conference
}

\author{
Fausto Roila • Jorn Herrstedt • Richard J. Gralla • \\ Maurizio Tonato
}

Received: 22 October 2010 /Accepted: 10 November 2010 /Published online: 28 December 2010

(C) Springer-Verlag 2010

Keywords MASCC antiemetic guidelines .

ESMO antiemetic guidelines

Despite the relevant progress achieved in the last 20 years, vomiting and, especially, nausea continue to be two of the most distressing side effects of cancer chemotherapy. In the late 1990 s, several professional organizations published recommendations on the optimal antiemetic prophylaxis in patients submitted to chemotherapy and radiotherapy.

Subsequently, due to the emergence of new findings and new antiemetic agents after the first recommenda-

\section{F. Roila}

Medical Oncology Division, Santa Maria Hospital,

Terni, Italy

J. Herrstedt ( $\square)$

Odense University Hospital,

Odense, Denmark

e-mail: herrstedt@ouh.regionsyddanmark.dk

\section{R. J. Gralla}

North Shore-LIJ Health System,

Hofstra University School of Medicine,

Lake Success,

New York, NY, USA

M. Tonato

Umbria Regional Cancer Network,

Perugia, Italy tions from 1997, representatives from several oncology societies met in Perugia, Italy in 2004 for a conference organized by the Multinational Association of Supportive Care in Cancer (MASCC) and updated the antiemetic guidelines [1]. Due to the publication of several new studies evaluating the role of the most recently developed antiemetic drugs, experts from the European Society of Medical Oncology (ESMO) and from MASCC thought it necessary to update the recommendations. To achieve this aim, the two societies organized on June 20-21, 2009 the third Consensus Conference on antiemetics in Perugia, Italy.

The methodology for the guideline process was based on a literature review through June 1, 2009 using MEDLINE (National Library of Medicine, Bethesda, MD, USA) and other databases, with evaluation of the evidence by an expert panel composed of 23 oncology professionals in clinical medicine, medical oncology, radiation oncology, surgical oncology, oncology nursing, statistics, pharmacy, pharmacology, medical policy, and decision making (Table 1). With the participating experts coming from ten different countries, on five continents, we believe that this is the most representative and evidence-based guideline process that has yet been performed.

The panel was comprised of ten committees dealing with major topics in this field (Table 2). Each committee was composed of five to seven members and each committee had one chair and co-chair. Each expert could be part of three or four committees but could only be a chair or cochair of one committee. During the consensus conference, 
Table 1 Participants in the antiemetic guideline process

Matti Aapro, Institut Multidisciplinaire d'Oncologie, Genolier, Switzerland

Enzo Ballatori, Medical Statistician, Via Pillino 2, Spinetoli, Italy

Emilio Bria, Regina Elena National Cancer Institute, Rome, Italy

Rebecca A. Clark-Snow, Lawrence Memorial Hospital, 325 Maine Street, Lawrence, Kansas, USA

Lawrence H. Einhorn, Walther Cancer Institute, Indianapolis, IN, USA

Birgitte T. Espersen, Aarhus University Hospital, Aarhus, Denmark

Petra Feyer, Clinic of Radiotherapy, Viventes Clinics, Berlin-Neukoelln, Berlin, Germany

Richard J. Gralla, North Shore-LIJ Health System, Hofstra University School of Medicine, Lake Success, New York, NY, USA

Steven M. Grunberg, University of Vermont, Burlington, VT, USA

Jørn Herrstedt, Odense University Hospital, Odense, Denmark

Paul J. Hesketh, Lahey Clinic, Burlington, MA, USA

Karin Jordan, University of Halle, Halle, Germany

Mark G. Kris, Memorial Sloan-Kettering Cancer Center, New York, NY, USA

Ernesto Maranzano, Radiation Oncology Centre, "S. Maria” Hospital, Terni, Italy

Alexander Molassiatis, School of Nursing, University of Manchester, Coupland, Manchester, UK

Gary Morrow, University of Rochester Cancer Center, Rochester, NY, USA

Ian Olver, Cancer Council Australia, Sydney, Australia

Bernardo L. Rapoport, The Medical Oncology Centre of Rosebank, Johannesburg, South Africa

Cynthia Rittenberg, Rittenberg Oncology Consulting, Metairie, LA, USA

Fausto Roila, Medical Oncology Division, Santa Maria Hospital, Terni, Italy

Mitsue Saito, Juntendo University Hospital, Tokyo, Japan

Maurizio Tonato, Umbria Regional Cancer Network, Perugia, Italy

David Warr, Princess Margaret Hospital, University of Toronto, Canada

the findings of each committee were presented by the chair to the entire expert panel. Although prevention of acute and delayed nausea and vomiting induced by highly- and moderately emetogenic chemotherapy had specific committees; at the end, these worked together as some of the issues are inseparable. Finally, the panel discussed the results and determined the level of evidence and the level of confidence for the recommendations according to ESMO and MASCC criteria [1,2].
To change the 2004 recommendations or for a new guideline recommendation to be accepted, a consensus of at least $66 \%$ of the expert panelists was needed. As a general rule, the panel considered changes of $10 \%$ or greater to be sufficient to warrant changing a guideline, given that the evidence supported this magnitude of benefit.

The results of the Perugia Conference are reported in this supplement of the Journal of Supportive Care in Cancer. Each article deals with one of the specific topics

Table 2 Antiemetic guidelines committees

\begin{tabular}{ll}
\hline I & Emetogenic classification of antineoplastic agents \\
II & Acute emesis: highly emetogenic chemotherapy \\
III & Delayed emesis: highly emetogenic chemotherapy \\
IV & Acute emesis: moderately emetogenic chemotherapy \\
V & Delayed emesis: moderately emetogenic chemotherapy \\
VI & Emesis induced by minimal or low emetogenic chemotherapy \\
VII & Additional issues: refractory emesis, rescue antiemetic therapy, multiple-day chemotherapy, high dose chemotherapy \\
VIII & Anticipatory emesis \\
IXa & Radiotherapy induced emesis \\
IXb & Antiemetics in children receiving chemotherapy \\
$\mathrm{X}$ & Future considerations: research directions, study design, economic considerations \\
\hline
\end{tabular}


reported in Table 2 and it has been written by the relative committee members who were actually participating in the Conference.

Acknowledgment To help organize the Consensus Conference, we received unrestricted grants from Eisai, Helsinn, GSK, Merck and Prostrakan. Additional funds were also committed by ESMO and MASCC.

\section{References}

1. Roila F, Hesketh PJ, Herrstedt J et al (2006) Prevention of chemotherapy- and radiotherapy-induced emesis: results of the 2004 Perugia International Antiemetic Consensus Conference. Ann Oncol 17:20-28

2. Herrstedt J, Roila F (2009) Chemotherapy-induced nausea and vomiting: ESMO clinical recommendations for prophylaxis. Ann Oncol 20(Suppl 4):156-158 